\title{
Designated path routing algorithm for dense wireless sensor network
}

\author{
Siva Kumar Subramaniam¹, Amierul Syazrul Azman², Mohamad Yusry Lee ${ }^{3}$, Farah Shahnaz Feroz ${ }^{4}$ \\ ${ }^{1}$ Advance Sensors and Embedded Controls System (ASECS), Fakulti Kejuruteraan Elektronik dan Kejuruteraan \\ Komputer, Universiti Teknikal Malaysia Melaka, Malaysia \\ ${ }^{2,3}$ Fakulti Kejuruteraan Elektronik dan Kejuruteraan Komputer, Universiti Teknikal Malaysia Melaka, Malaysia \\ ${ }^{4}$ Pervasive Computing and Educational Technology (PET), Fakulti Kejuruteraan Elektronik dan Kejuruteraan Komputer, \\ Universiti Teknikal Malaysia Melaka, Malaysia
}

\section{Article Info}

Article history:

Received Jan 15, 2021

Revised Jun 30, 2021

Accepted Jul 7, 2021

\section{Keywords:}

Grid

Oil and gas

Routing protocol

Wireless sensor network

WSN

\begin{abstract}
Due to extensive pipeline dissemination in the oil and gas refinery, the nodes need to be placed in a grid formation. As such, since most oil and gas industry applications require continuous data gathering, a heavy data stream will be introduced in the network traffic, mainly when the network density is high. As a result, performance degradation and poor energy consumption will occur. Ad hoc on-demand distance vector and optimized link state routing protocol have been simulated to investigate these issues further. Due to packet congestion, the network experiences a domino effect on the performance, such as packet loss, throughput degradation, and poor energy consumption. Thus, a tailored solution is required since oil and gas industry relies heavily on sensor data to keep track of pipelines condition to prevent anomalous events from happening. The proposed algorithm has been developed to optimize the network performance by dividing the traffic into two and by reducing the flooding during route discovery. The results have shown better network performance and energy consumption can be achieved using the proposed algorithm when compared to the others.
\end{abstract}

This is an open access article under the CC BY-SA license.

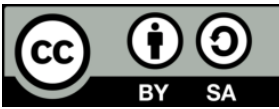

\section{Corresponding Author:}

Siva Kumar Subramaniam

Advance Sensors and Embedded Controls System (ASECS)

Fakulti Kejuruteraan Elektronik dan Kejuruteraan Komputer

Universiti Teknikal Malaysia Melaka, Malaysia

76100 Durian Tunggal, Malacca, Malaysia

Email: siva@utem.edu.my

\section{INTRODUCTION}

In oil and gas industry, the extraction of materials from underground or underwater begins at the upstream stage as shown in Figure 1 [1]. These processed materials are then transported to the downstream stage via pipelines, barges, or tank trucks. End-products are manufactured in the downstream stage through a series of processing and refining [2]. The pipeline is the cheapest and most reasonable transportation in oil and gas industry [3]. However, due to the surrounding environment, the pipelines are exposed to corrosion, leakage, or unstable pressure. Since the pipelines carry harsh materials, any deformation could cause an explosion that threatens both environmental and financial. Thus, constant monitoring is required to ensure a safer pipeline operation can be achieved for an extended period.

Wireless sensor network (WSN) has been used in oil and gas pipeline condition monitoring since the sensor data can be transmitted wirelessly, particularly in a harsh location where human intervention is highly considered [4]. WSN also has been used in many applications such as agriculture monitoring, smart 
city, and military surveillance [5], [6]. WSN refers to a group of sensor nodes communicating with each other to ensure the sensed data can be received by the base station wirelessly [7]. Each sensor node is made up of several sensors, controller, power component, and transceiver module. Since most oil and gas applications require continuous data gathering, a substantial amount of packets will accumulate in the traffic, particularly when the network size is large [8].

This paper aims to highlight the network performance issues when the network size varies. The next aim of this paper is to propose an optimized routing algorithm for oil and gas pipeline condition monitoring application. This paper focuses on optimizing network performance on the network layer of open systems interconnection (OSI) model in accordance with institute of Electrical and Electronics Engineers (IEEE) 802.11 standard for refinery pipeline condition monitoring application [9]. The nodes are arranged in a scattered manner to imitate the actual deployment in the refinery pipeline and to achieve thorough communication coverage. The novelties of this paper include (1) issuing the initial findings on the network performance using grid node arrangement when the network size varies since there is no study conducted on the grid node arrangement in accordance with IEEE 802.11 standard. The novelties of this paper also include (2) presenting the technique used in the proposed algorithm in regard to the findings in (1).

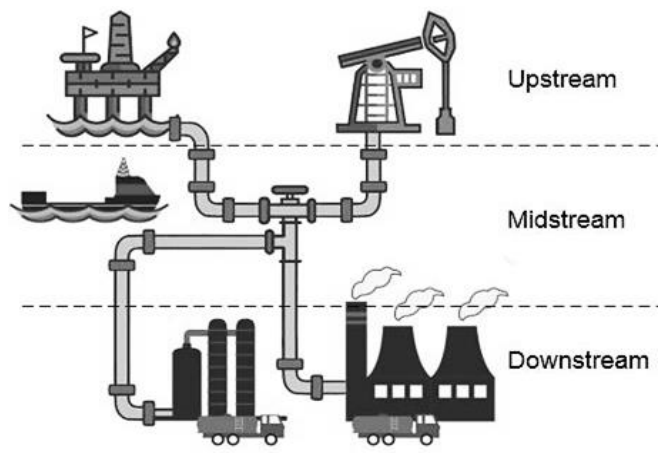

Figure 1. Three main stages in oil and gas industry

\section{PROBLEM STATEMENTS}

According to the survey done by the researchers in [1], there are four most prominent challenges of WSNs in oil and gas applications, which are reliability, scalability, robustness, and energy consumption. A reliable network is a network that can deliver performance reasonably, while a scalable network is a network that can preserve its performance when the loads increase [10]. Robustness determines the strength of the network in maintaining its communication link against nodes failure, interference, or a security attack. In any communication network, security has always been an issue, including wormhole attack, eavesdropping, and signal jamming. Managing energy consumption is also very important to prevent the nodes to die since the maintenance task in oil and gas pipeline area is extremely risky [11], [12].

Since oil and gas applications require continuous data gathering to prevent anomalous events from happening, the network suffers from packet loss due to congestion in the traffic [13]. In a multi-hop network, the nodes accumulate packets from the previous forwarder in a direction towards the destination node [14]. The packets that enqueue in the queue include data and control packets. Once the queue is fully occupied, the next packet will be dropped and the network will experience packet loss and throughput degradation [15]. Additional energy will be consumed when the source nodes regenerate the dropped packet. Also, due to the accumulation factor, the nodes closer to the destination node will have to handle more packets and consume more resources than the nodes that are further from the destination node [16]. This event is known as an unfair state of the network [17].

\section{BACKGROUND WORKS}

The network layer handles the packet routing and forwarding between the sensor nodes in a network according to the implemented routing protocol. Reactive routing protocol uses an ad-hoc approach where the route discovery is invoked only when needed [18]. This feature allows the protocol to produce a reduced amount of routing overhead which helps in conserving the network resources [19], [20]. However, data forwarding will be delayed due to the time-consuming process of route discovery. Ad-hoc on-demand distance vector (AODV) routing protocol is an example of reactive routing protocols [21]. 
As compared to reactive routing protocols, the proactive routing protocol is deemed as a heavier protocol since it periodically updates the routing table, which produces a substantial amount of routing overhead and control packet [22]. This feature helps the data to be sent in a timely manner since all the routing information is readily available [23]. The proactive routing protocol is also known as the table-driven routing protocol. Optimized link state routing (OLSR) an example of proactive routing protocol [24].

Hybrid routing protocol combines the good features in reactive and proactive routing protocol [20], [25]. Zone routing protocol (ZRP) is an example of hybrid routing protocol that has been developed to reduce the overconsumption of network resources and to optimize the delay during data delivery [26]. ZRP routing protocol is not covered in this paper since it uses two-tier routing architecture. Certainly, a high amount of generated packets due to the continuous monitoring and the high number of nodes can contribute to the traffic congestion regardless of what type of routing protocol is implemented as shown in (1).

$$
P_{t}=\left[\left(P_{\alpha}\right)+\sum_{\beta=\alpha+1}^{n_{0}}\left(P_{\beta}\right)\right] \leq Q
$$

Where $n_{0}=N-1$ and $N$ is the total number of nodes in the network. $P_{t}$ is the total amount of packets for the whole network and $P_{\alpha}$ is the amount of data and control packets at intermediate node $\alpha . P_{\beta}$ is the amount of data and control packets at the rest of the node $\beta$. It can be seen that the traffic will overflow if the number of packets produced is beyond the queue limit. Such an event can contribute to performance degradation as discussed earlier. Motivated by this observation, a routing algorithm has been proposed in the next section.

\section{DUAL DESIGNATED PATH ROUTING TECHNIQUE}

Odd-even for grid (OEG) routing algorithm divides the network into two dedicated traffics; odd traffic and even traffic. In general, a route is discovered according to the freshness and the shortest path to the destination. However, OEG routing algorithm selects the route according to the determination of the internet protocol (IP) address on the nodes with the consideration of both $\mathrm{x}$ and $\mathrm{y}$-axis of the network. Each oddnumbered node belongs to odd traffic and each even-numbered node belongs to even traffic.

In the route discovery process in the forward direction, a source sends route request (RREQ) packets towards its neighboring nodes according to the odd-even determination as shown in Figure 2. If the source is odd-numbered, only odd-numbered neighboring nodes will receive the RREQ packets. Else if the source is even-numbered, only even-numbered neighboring nodes will receive the RREQ packets. The odd-even determination is performed for each RREQ forwarding between the respective nodes. Once the RREQ packets arrived at the destination node, a route reply (RREP) packet is issued to the source node by the destination node. During this period, the nodes that act as RREP forwarders are the same as in RREQ forwarding but in the reverse direction. Once the RREP arrived at the source, the data packet now can be sent to the destination node using the established route.

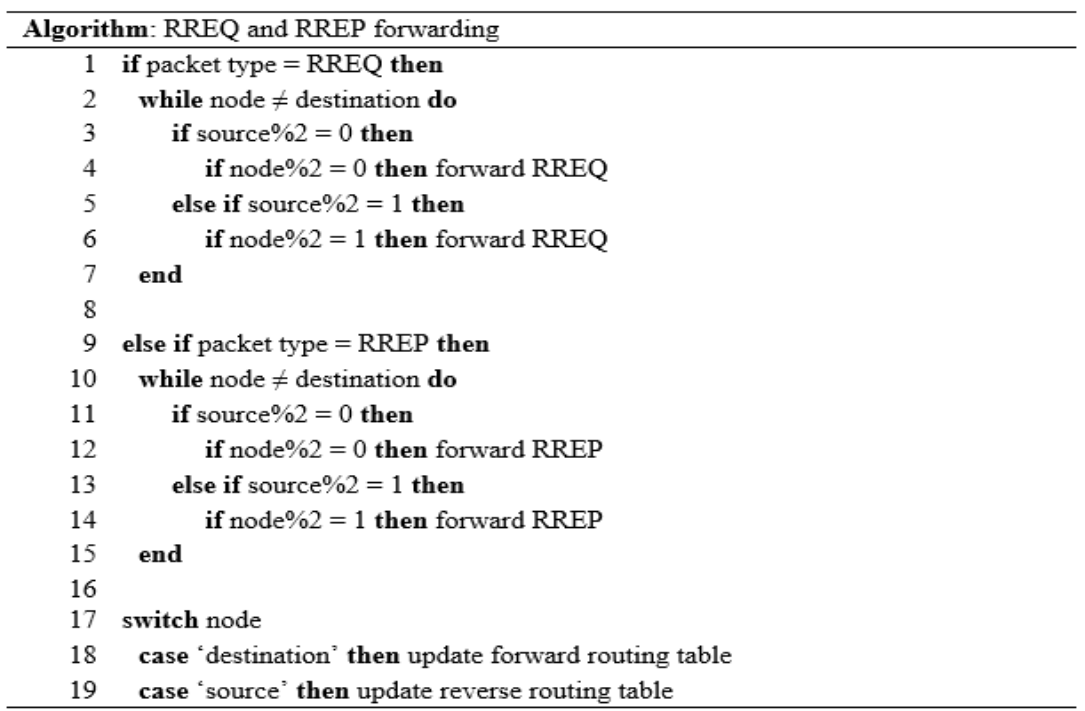

Figure 2. OEG routing algorithm 
By using OEG routing algorithm, the packet accumulation and packet contention can be reduced since the traffic has been divided into two as shown in Figure 3. The distance between nodes is represented as $d$. In real life situation, this feature can be seen in roadway traffic where a two-lane roadway offers a lower chance of congestion occurrence as compared to a one-lane roadway. The traffic division can be represented as in (6).

$$
\begin{aligned}
& P_{e}=\left[\left(P_{2 \alpha}\right)+\sum_{\beta=\alpha+1}^{n_{1}}\left(P_{2 \beta}\right)\right] \leq Q_{e} \\
& n_{1}=\left\{\begin{array}{c}
\frac{N n}{2}, \text { if } N n \text { is even } \\
\frac{N n-1}{2}, \text { else if } N n \text { is odd }
\end{array}\right. \\
& P_{o}=\left[\left(P_{2 \alpha+1}\right)+\sum_{\beta=\alpha+1}^{n_{2}}\left(P_{2 \beta+1}\right)\right] \leq Q_{o} \\
& n_{2}=N n-n_{1}-1 \\
& P_{T}=P_{e}+P_{o} \leq Q
\end{aligned}
$$
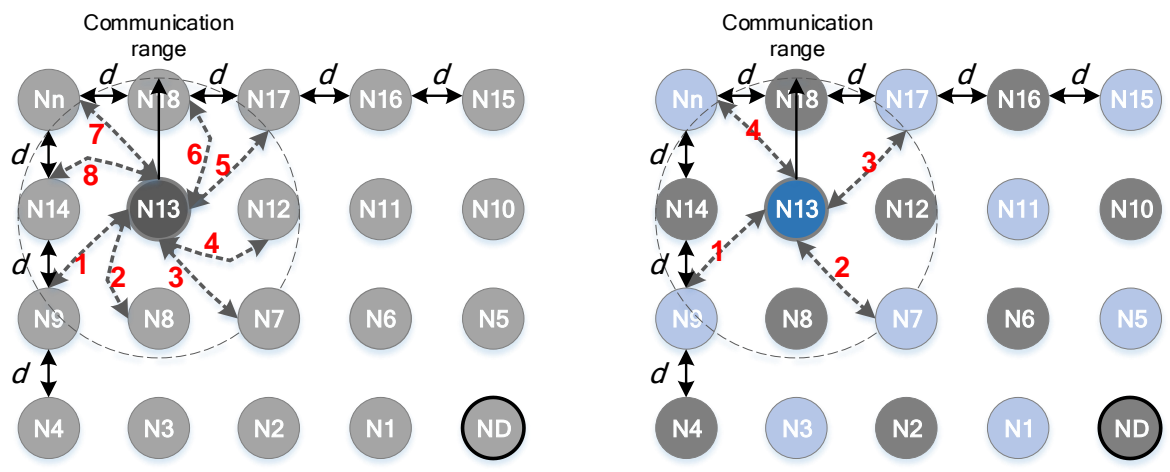

Figure 3. Broadcasting in conventional routing (left) and OEG routing (right)

$P_{e}$ and $P_{o}$ is the total number of packets in even and odd traffic respectively. $P_{2 \alpha}$ and $P_{2 \alpha+1}$ is the total number of packets (control and data packets) at the intermediate node with even and odd address respectively. $P_{2 \beta}$ and $P_{2 \beta+1}$ is the total number of packets (control and data packets) at the rest of the nodes with even and odd address respectively. $Q_{e}$ and $Q_{o}$ is the interface queue limit for even and odd traffic respectively. The total number of packets for the whole network is $P_{T}$.

Apart from that, OEG algorithm reduces the number of broadcast packets since the RREQ forwarding has been reduced to half as shown in Figure 3. This feature is important to optimize the space in the queue and the consumption of network resources. In addition, the number of possible forwarders for each traffic is $50 \%$ of the whole network (traffic division). In total, the number of broadcast packets for each traffic has been reduced to one-fourth fewer as compared to conventional routing. In (7) represents the total number of broadcast packets in conventional routing and in (9) represents the total number of broadcast packets in OEG routing for each traffic.

$$
\begin{aligned}
& N_{b}=m \times n=m n \\
& m_{e}=m_{o}=\frac{m}{2} \\
& N_{b e}=N_{b o}=\frac{m}{2} \times \frac{n}{2}=\frac{m n}{4}
\end{aligned}
$$

$N_{b}$ is the total number of broadcast packets in conventional routing, $m$ is the total number of possible forwarders, and $n$ is the total number of broadcasting for each node. In OEG routing, $m_{e}$ and $m_{o}$ is the total number of possible forwarders in even and odd traffic respectively. Each traffic has $50 \%$ number of possible forwarders in the network. $N_{b e}$ and $N_{b o}$ is the total number of broadcast packets in even and odd 
traffic respectively. A number of simulations have been conducted to investigate the impact of the traffic division and the reduction of the number of broadcast packets on the network performance.

\section{SIMULATION SETUP}

The simulated routing algorithms are AODV, OLSR, and OEG. The simulation time is 500 seconds and the distance between each node, $d$, is 50 meters to simulate the actual deployment in most of the pipeline applications. The transmission rate is one packet every 2 seconds. The rest of the simulation parameters are as listed in Table 1. The results were averaged from the five best out of seven randomly generated scenarios to achieve a detailed performance evaluation.

Table 1. Simulation parameters using network simulator 2.35

\begin{tabular}{ll}
\hline \multicolumn{1}{c}{ Parameters } & \multicolumn{1}{c}{ Value } \\
\hline Number of nodes & $24,48,80,120,168,224,288$, and 360 \\
Packet size & 128 bytes \\
Interface queue type & DropTail/PriQueue \\
Packet queue length & 50 \\
MAC & IEEE 802.11 \\
Traffic type & CBR \\
Propagation model & Two ray ground \\
Number of nodes & $24,48,80,120,168,224,288$, and 360 \\
\hline
\end{tabular}

\section{RESULTS AND DISCUSSION}

\subsection{Packet delivery ratio and throughput}

Packet delivery ratio is important in designing a network since it helps to identify the issues that happen in a network. The packet delivery ratio shows how many packets have been successfully received by the destination over the sent packets. Since oil and gas applications are data-driven, minimizing the number of packet loss as low as possible is very important to the industry to ensure the authorities able to keep track of the assets condition (pipeline). Figure 4 shows OEG algorithm outperforms AODV and OLSR starting from 168 nodes onwards. Also, due to the packet loss starting at 120 nodes onwards, it can be seen that the achieved throughput also has degraded. However, OEG outperforms AODV and OLSR in achieving better throughput performance.

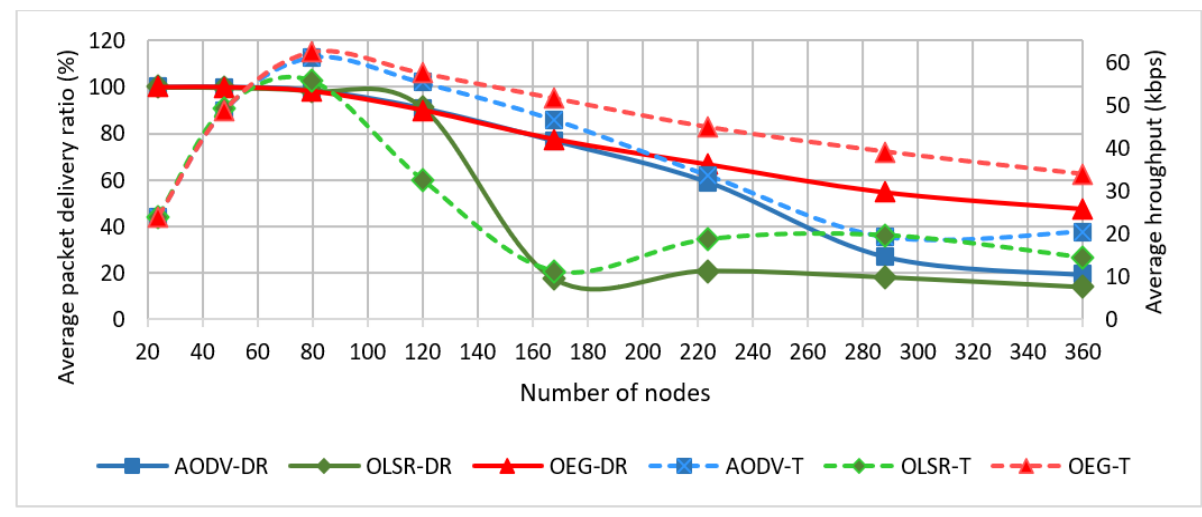

Figure 4. Packet delivery ratio and throughput against number of nodes

\subsection{Energy consumption}

Due to the harsh environment in the refinery pipeline area, reducing human intervention is extremely beneficial in oil and gas industry. Each sensor node is powered by a battery pack and once the node died, the maintenance workers need to replace the battery pack. Such an event shows that the optimization of energy consumption in the network is crucial. A substantial amount of energy is wasted due to the regeneration and reforwarding of packets due to packet loss. As shown in Figure 5, OEG algorithm shows the fewest energy consumption starting from 120 nodes onwards. As the number of nodes increase, the number of packet forwarding also increases. Hence, the amount of energy consumption also increases. 


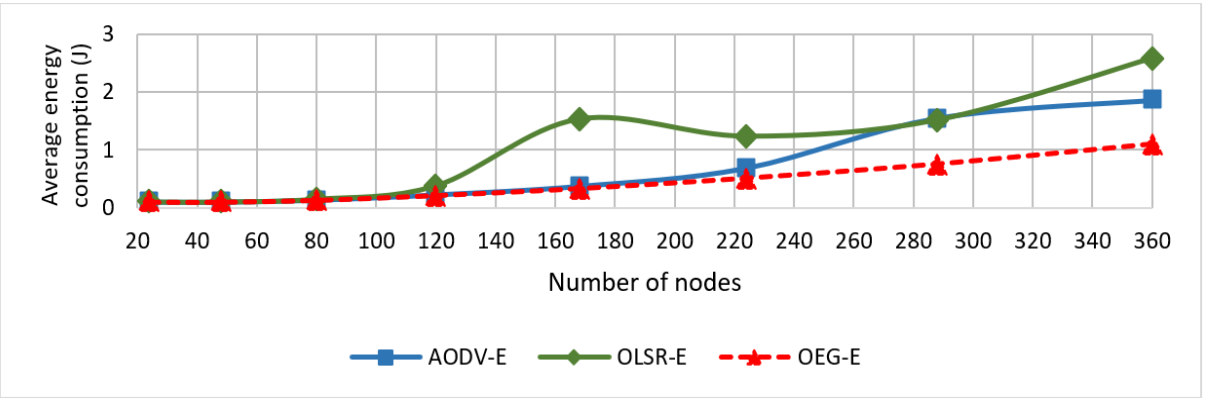

Figure 5. Energy consumption against number of nodes

\subsection{Fairness index and passive node}

The nodes that are closer to the destination accumulate a higher number of packets as compared to the nodes that are further from the destination. This event shows that the network resources were not equally distributed by the nodes (unfair state) in the network. As a result, some nodes will become passive nodes. Passive node describes the inability of the node to transmit its packet due to the unavailability of network resources. The network resources have been wasted due to the excessive flooding of control packets and overhead.

Jain's fairness index is used to measure the fairness of the network in this study. In Figure 6, OLSR shows the worst fairness index and OEG shows the best fairness index starting at 80 nodes onwards. By reducing the number of broadcast packets, OEG able to reduce the number of passive nodes significantly starting at 120 nodes onwards. OEG shows the fewest number of passive nodes present in the network.

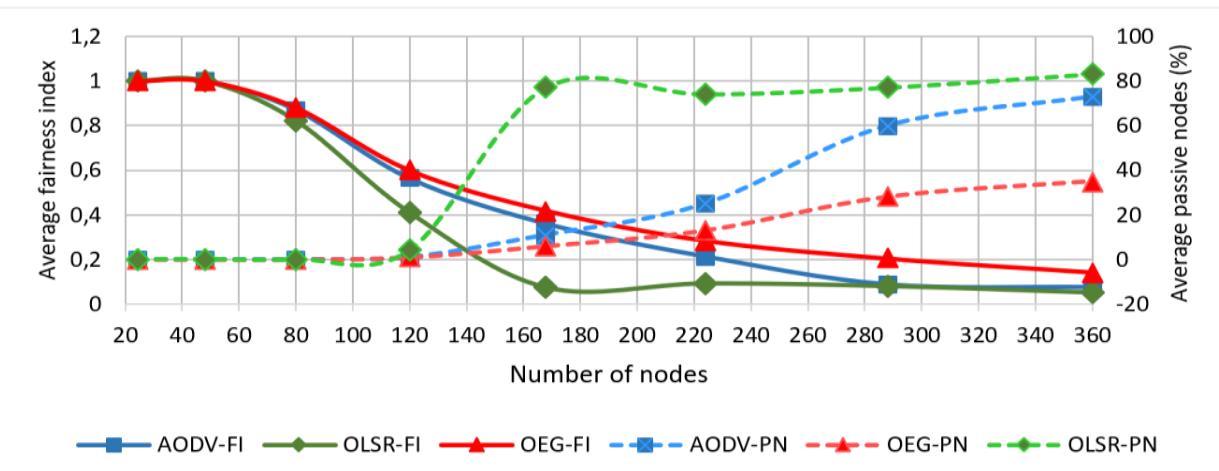

Figure 6. Fairness index and number of passive nodes against number of nodes

\section{CONCLUSION}

In oil and gas refinery pipeline condition monitoring, deployment of a vast amount of sensor nodes is required to ensure thorough communication coverage for the entire pipeline area can be achieved. However, in addition to the continuous data gathering of the application, such a deployment contributes to network congestion, which will lead to performance degradation and poor energy consumption. Performance optimization is important since most of the applications in oil and gas industry are data-driven. Hence, any data loss can cause the authorities to fail to keep track of the condition of the pipeline. A tailored routing algorithm has been proposed to enhance the network performance by minimizing the packet accumulation via traffic splitting and by reducing the number of broadcast packets. The proposed algorithm showed a significant improvement on packet delivery ratio, throughput, energy consumption, and passive nodes. However, fairness is highlighted as the weakness of the proposed algorithm since there is only a slight improvement can be seen on the fairness index in the results.

\section{ACKNOWLEDGEMENTS}

The authors would like to thank the Ministry of Higher Education-Malaysia, Universiti Teknikal Malaysia-Melaka for their support, Lab facilities, sincere encouragement, and assistance. 


\section{REFERENCES}

[1] M. Y. Aalsalem, W. Z. Khan, W. Gharibi, M. K. Khan, and Q. Arshad, "Wireless Sensor Networks in oil and gas industry: Recent advances, taxonomy, requirements, and open challenges," Journal of network and computer applications, vol. 113, pp. 87-97, July 2018, doi: 10.1016/j.jnca.2018.04.004.

[2] W. Z. Khan, M. Y. Aalsalem, M. K. Khan, M. S. Hossain and M. Atiquzzaman, "A reliable Internet of Things based architecture for oil and gas industry," 2017 19th International Conference on Advanced Communication Technology (ICACT), 2017, pp. 705-710, doi: 10.23919/ICACT.2017.7890184.

[3] M. Z. Abbas, K. A. Baker, M. Ayaz, and H. Mohamed, "Key Factors Involved in Pipeline Monitoring Techniques Using Robots and WSNs: Comprehensive Survey," Journal of Pipeline Systems Engineering and Practice, vol. 9 , no. 2, p. 04018001, 2018.

[4] N. F. Henry and O. N. Henry, "Wireless Sensor Networks based Pipeline Vandalisation and Oil Spillage Monitoring and Detection: Main Benefits for Nigeria Oil and Gas Sectors," The SIJ Transactions on Computer Science Engineering \& its Applications (CSEA), vol. 3, no. 1, pp. 1-6, 2015.

[5] A. Goswami and M. Kumar, "A Review on Energy Harvesting in Wireless Sensor Networks," Int. J. Innov. Res. Sci. Eng. Technol., vol. 6, no. 4, pp. 6863-6867, 2017.

[6] G. Han, J. Jiang, N. Bao, L. Wan, and M. Guizani, "Routing protocols for underwater wireless sensor networks," in IEEE Communications Magazine, vol. 53, no. 11, pp. 72-78, November 2015, doi: 10.1109/MCOM.2015.7321974.

[7] M. Raza, N. Aslam, H. Le-Minh, S. Hussain, Y. Cao, and N. M. Khan, "A Critical Analysis of Research Potential, Challenges, and Future Directives in Industrial Wireless Sensor Networks," in IEEE Communications Surveys \& Tutorials, vol. 20, no. 1, pp. 39-95, Firstquarter 2018, doi: 10.1109/COMST.2017.2759725.

[8] M. A. Kafi, D. Djenouri, J. Ben-Othman, and N. Badache, "Congestion Control Protocols in Wireless Sensor Networks: A Survey," in IEEE Communications Surveys \& Tutorials, vol. 16, no. 3, pp. 1369-1390, Third Quarter 2014, doi: 10.1109/SURV.2014.021714.00123.

[9] IEEE 802.11 Working Group, "Specific requirements Part 11: Wireless LAN Medium Access Control (MAC) and Physical Layer (PHY) Specifications," ANSI/IEEE Std. 802.11-1999, 1999.

[10] T. P. Raptis, A. Passarella, and M. Conti, "Data Management in Industry 4.0: State of the Art and Open Challenges," in IEEE Access, vol. 7, pp. 97052-97093, 2019, doi: 10.1109/ACCESS.2019.2929296.

[11] B. A. Sundaram, K. Kesavan, and S. Parivallal, "Recent Advances in Health Monitoring and Assessment of In-Service Oil and Gas Buried Pipelines," J. Inst. Eng. India Ser. A, vol. 99, no. 4, pp. 729-740, 2018, doi: 10.1007/s40030-018-0316-5.

[12] X. Fu, H. Yao, O. Postolache, and Y. Yang, "Message forwarding for WSN-Assisted Opportunistic Network in disaster scenarios," Journal of Network and Computer Applications, vol. 137, pp. 11-24, July 2019, doi: 10.1016/j.jnca.2019.04.005.

[13] M. Hammoudeh and R. Newman, "Adaptive routing in wireless sensor networks: QoS optimization for enhanced application performance," Information Fusion, vol. 22, pp. 3-15, March 2013, doi: 10.1016/j.inffus.2013.02.005.

[14] M. T. Abbas, A. Muhammad, and W. C. Song, "SD-IoV: SDN Enabled Routing for Internet of Vehicles in RoadAware Approach," Journal of Ambient Intelligence and Humanized Computing, vol. 11, no. 3, pp. 1265-1280, March 2020, doi: 10.1007/s12652-019-01319-w.

[15] M. Baklizi, "Weight Queue Dynamic Active Queue Management Algorithm,” Symmetry, vol. 12, no. 12, p. 2077, December 2020, doi: 10.3390/sym12122077.

[16] K. Jaiswal, and V. Anand, "EOMR: An energy-efficient optimal multi-path routing protocol to improve QoS in wireless sensor network for IoT applications," Wireless Personal Communications, pp. 1-23, 2019, doi: 10.1007/s11277-019-07000-x.

[17] A. Ahmad, M. Beg, and S. Ahmad, "Fairness issues and measures in wireless networks: A survey," IOSR Journal of Electronics and Communication Engineering, vol. 11, no. 6, pp. 20-24, 2016, doi: 10.9790/2834-1106042024.

[18] S. K. Singh, R. Duvvuru, and J. P. Singh, "TCP and UDP-based performance evaluation of proactive and reactive routing protocols using mobility models in MANETS," International Journal of Information and Communication Technology, vol. 7, no. 6, pp. 632-644, 2015, doi: 10.1504/IJICT.2015.072043.

[19] A. K. Yadav and A. Kush, "TCP- and UDP-Based Performance Evaluation of AODV and DSR Routing Protocol on Varying Speed and Pause Time in Mobile Ad Hoc Networks," in Next-Generation Networks Springer, Singapore, vol. 638, pp. 323-332, 2018, doi: 10.1007/978-981-10-6005-2_34.

[20] I. Y. Mohammed, "Comparative analysis of proactive \& reactive protocols for cluster based routing algorithms in WSNs," Wold Sci. News an Int. Sci. J., vol. 124, no. 2, pp. 131-142, 2019.

[21] S. Goswami, S. Joardar, C. B. Das, S. Kar, and D. K. Pal, "Performance Analysis of Three Routing Protocols in MANET Using the NS-2 and ANOVA Test with Varying Speed of Nodes," in Ad Hoc Networks, 2017, pp. 126138, doi: 10.5772/66521.

[22] H. Xin and K. Yang, "Routing Protocols Analysis for Internet of Things," 2015 2nd International Conference on Information Science and Control Engineering, 2015, pp. 447-450, doi: 10.1109/ICISCE.2015.104.

[23] V. Jigisha, C. S. Raju, and C. Balaswamy, "The comparison between OLSR and AODV routing protocols for Vehicular Adhoc Networks," Int. J. Adv. Res. Comput. Commun. Eng., vol. 4, no. 3, pp. 467-470, 2015, doi: 10.17148/IJARCCE.2015.43112.

[24] T. Clausen, C. Dearlove, P. Jacquet, and U. Herberg, "The optimized link state routing protocol version 2," Internet Eng. Task Force, pp. 1-115, 2014.

[25] J. Govindasamy and S. Punniakody, "A comparative study of reactive, proactive and hybrid routing protocol in wireless sensor network under wormhole attack," Journal of Electrical Systems and Information Technology, vol. 5, no. 3, pp. 735-744, December 2018, doi: 10.1016/j.jesit.2017.02.002.

[26] Y. M. Rao, M. V Subramanyam, and K. S. Prasad, "Cluster Based Hybrid Routing Protocol for Wireless Mesh Networks,” Wirel. Pers. Commun., vol. 103, no. 4, pp. 3009-3023, 2018, doi: 10.1007/s11277-018-5990-0. 